\title{
Methane mitigation potential of phyto-sources from Northeast India and their effect on rumen fermentation characteristics and protozoa in vitro
}

\author{
Luna Baruah ${ }^{1,2}$, Pradeep Kumar Malik ${ }^{1}$, Atul P. Kolte ${ }^{1}$, Arindam Dhali ${ }^{1}$ and Raghavendra Bhatta
}

1. Energy Metabolism Laboratory, ICAR-National Institute of Animal Nutrition and Physiology, Bengaluru - 560030 , Karnataka, India; 2. Faculty of Biotechnology, Jain University, Bengaluru, Karnataka, India. 3. ICAR-National Institute of Animal Nutrition and Physiology, Bengaluru - 560 030, Karnataka, India.

Corresponding author: Pradeep Kumar Malik, e-mail: malikndri@gmail.com

Co-authors: LB: luna.baruah09@gmail.com, APK: atulkolte@gmail.com, AD: dhali72@gmail.com, RB: ragha0209@yahoo.com

Received: 23-02-2018, Accepted: 01-05-2018, Published online: 19-06-2018

doi: 10.14202/vetworld.2018.809-818 How to cite this article: Baruah L, Malik PK, Kolte AP, Dhali A, Bhatta R (2018) Methane mitigation potential of phyto-sources from Northeast India and their effect on rumen fermentation characteristics and protozoa in vitro, Veterinary World, 11(6): 809-818.

\begin{abstract}
Aim: The aim of the study was to explore the anti-methanogenic potential of phyto-sources from Northeast region of the country and assess the effect on rumen fermentation characteristics and protozoa for their likely inclusion in animal diet to reduce methane emission.

Materials and Methods: Twenty phyto-sources were collected from Northeast state, Assam, during March to April 2014. Phyto-sources were analyzed for their tannin content followed by screening for methane mitigation potential using in vitro system. The effect of tannin on methane production and other fermentation parameters was confirmed by attenuating the effect of tannin with polyethylene glycol (PEG)-6000 addition. About $200 \mathrm{mg}$ dried phyto-source samples were incubated for $24 \mathrm{~h}$ in vitro, and volume of gas produced was recorded. The gas sample was analyzed on gas chromatograph for the proportion of methane in the sample. The effect of phyto-sources on rumen fermentation characteristics and protozoal population was determined using standard methodologies.
\end{abstract}

Results: Results from studies demonstrated that Litchi chinensis, Melastoma malabathricum, Lagerstroemia speciosa, Terminalia chebula, and Syzygium cumini produced comparatively less methane, while Christella parasitica, Leucas linifolia, Citrus grandis, and Aquilaria malaccensis produced relatively more methane during in vitro incubation. An increase $(\mathrm{p}<0.05)$ in gas and methane production from the phyto-sources was observed when incubated with PEG-6000. Entodinimorphs were prominent ciliates irrespective of the phyto-sources, while holotrichs represented only small fraction of protozoa. An increase $(\mathrm{p}<0.05)$ in total protozoa, entodinimorphs, and holotrichs was noted when PEG-6000 added to the basal substrate. Our study confirmed variable impact of phyto-sources on total volatile fatty acid production and ammonia-N.

Conclusion: It may be concluded that L. chinensis, M. malabathricum, L. speciosa, S. cumini, and T. chebula are having potent methane suppressing properties as observed in vitro in $24 \mathrm{~h}$. These leaves could be supplemented in the animal diet for reducing methane emission; however, in vivo trials are warranted to confirm the methane inhibitory action and optimize the level of supplementation.

Keywords: methane, phyto-sources, protozoa, rumen fermentation, tannin.

\section{Introduction}

Rumen methanogenesis is an integral mechanism associated with complex carbohydrate metabolism for the removal of fermentative gases. The removal of fermentative gases particularly carbon dioxide and hydrogen is prerequisite to keep the redox potential within the threshold limit. On the contrary, rumen methanogenesis invariably contributes to the global warming apart from depriving the host animal to a significant fraction of biological energy. The energy loss due to enteric methane emission varies between $2 \%$ and $12 \%$ depending on

Copyright: Baruah, et al. Open Access. This article is distributed under the terms of the Creative Commons Attribution 4.0 International License (http://creativecommons.org/licenses/by/4.0/), which permits unrestricted use, distribution, and reproduction in any medium, provided you give appropriate credit to the original author(s) and the source, provide a link to the Creative Commons license, and indicate if changes were made. The Creative Commons Public Domain Dedication waiver (http://creativecommons.org/ publicdomain/zero/1.0/) applies to the data made available in this article, unless otherwise stated. the feed type and their digestibility [1]. The host animal is deprived for $39.5 \mathrm{KJ}$ energy for each liter of enteric methane emission [2]. About $90 \mathrm{Tg}$ enteric methane is added per year to the global atmospheric pool [3], and $11 \%$ of the global enteric methane emission is contributed by the Indian livestock [4]. The acute shortage of quality feed and fodders in the country and feeding primarily on high fiber diet are few of the obvious reasons for large methane emission from Indian livestock. Feeding of ruminants on quality feed, i.e., succulent fodder and concentrate is probably the best approach for achieving methane reduction, but factors such as increasing human population, limited land allocation under fodder production, and food-feed-fuel competition pose a check on the use of high-quality feeds for feeding purpose. Further, the use of ionophores such as monensin and lasalocid as feed additives and methane suppressant has been banned in many countries, and others are also in process of following the same. 
Therefore, it has become a necessity for the animal nutritionist to search for new feed resources which could be used with dietary ingredients for the purpose of methane mitigation. The anti-methanogenic properties of phyto-sources are usually due to the secondary metabolites which are integral component and serve as first line of defense against insect and pest. Tannins, saponin, and essential oil are few important plant secondary metabolites; however, their anti-methanogenic properties have recently been explored [5-7] and tannin is largely studied plant secondary metabolites among all. However, there has been a variation in tannin's activity from different sources and all the phyto-sources are not equally effective in achieving the methane reduction even though they possess similar tannin content [6]. Further, the phyto-source available in one geographical location may not be available in farther most located area $[6,8,9]$. Hence, we should keep broadening the list of phyto-sources that contain measurable tannin content and promising for methane reduction. Diverse kind of phyto-sources is abundant in Northeast state, Assam, which shared Himalayan and Indo-Burmese biodiversity hotspot [10]; however, these sources never been explored for their anti-methanogenic properties.

Thus, the present study was undertaken to investigate the anti-methanogenic potential of 20 medicinal phyto-sources abundantly available in Northeast India.

\section{Materials and Methods}

\section{Ethical approval}

As the study was in vitro and not conducted on live animals; hence, no ethical approval was required for the study.

\section{Sample collection}

A total of 20 phyto-samples (leaves) known for their ethnomedicinal properties were collected from the Northeastern state, Assam $\left(26.2006^{\circ} \mathrm{N}\right.$ and $92.9376^{\circ} \mathrm{E}$ ), India, during March to April 2014. All the plants were in their reproductive stage (capable of producing reproductive parts). Approximately $500 \mathrm{~g}$ of fresh leaves (both mature and immature) were harvested from the tree and air-dried for 10 days. The air-dried samples were brought to the Energy Metabolism Laboratory of the National Institute of Animal Nutrition and Physiology, Bengaluru, and ground samples to $1 \mathrm{~mm}$ size using Cyclotec Mill for further analysis. The ground samples were preserved in airtight container and stored after labeling in dark place to prevent the degradation of phenolics.

\section{Chemical composition}

The leaves sample were analyzed in triplicate for crude protein $(\mathrm{CP})$ using standard procedure [11]; while neutral detergent fiber (NDF) and acid detergent fiber (ADF) of leaves were estimated following Van Soest et al. [12] methodology. The tannin content in leaves was determined as per a study of Makkar [13].
Briefly, $0.2 \mathrm{~g}$ ground sample was extracted in $10 \mathrm{ml}$ aqueous acetone (acetone-water, 7:3) for $20 \mathrm{~min}$ in an ultrasonic water bath. The extraction was repeated twice, and the extract was centrifuged $(6000 \mathrm{~g})$ for $10 \mathrm{~min}$ at $4^{\circ} \mathrm{C}$. The supernatant was combined and used for tannin analysis on the same day. Total phenol (TP) and total tannin (TT) in samples were assessed by modified Folin-Ciocalteu method [13] using polyvinylpolypyrrolidone and expressed as gallic acid equivalents. However, condensed tannin (CT) in sample was analyzed by butanol-HCl-iron method [13] and expressed as leucocyanidin equivalents. The hydrolysable tannin (HT) was calculated by difference of TT and CT [6].

\section{In vitro incubation and gas analysis}

For in vitro studies, rumen liquor was collected at $9.00 \mathrm{~h}$ from two cannulated Holstein-Friesian crossbred bulls $(330 \mathrm{~kg})$ before morning feeding. The cannulated animals were fed on a total mixed ration comprising finger millet (Eleusine coracana) straw and commercial concentrate mixture mixed in the ratio of 70:30. The rumen liquor collected was strained through muslin cloth prior adding to the buffer (bicarbonate, mineral, and distill water) prepared as per Menke et al. [14]. This was followed by incubation of air-dried $200 \mathrm{mg}$ sample along with $30 \mathrm{ml}$ buffered rumen inoculum in $100 \mathrm{ml}$ calibrated glass syringe placed in a water bath shaker at $39^{\circ} \mathrm{C}$. All samples were incubated 2 consecutive times, wherein three replicates of each samples were used in individual incubation. For every set of incubation and to nullify the effect of rumen inoculum, three syringes were kept as blank (without sample). Individual incubation was set up with three replicates of standard hay and concentrate from Hohenheim University as positive control [14]. Fermentation was terminated on the completion of $24 \mathrm{~h}$. The gas production was calculated by difference of final and initial piston position and considering the gas production from blank syringes which contains no inoculum. The effect of tannin on methane production and rumen fermentation was confirmed by incubating the samples with polyethylene glycol (PEG)-6000 in the ratio of 1:2. Gas samples were collected in evacuated glass vials and $1 \mathrm{~mL}$ was withdrawn with the help of Hamilton Syringe for the gas chromatograph analysis. Methane was estimated using gas chromatograph (Chemito GC-1000, India) equipped with thermal conductivity detector and Porapak-Q column. Injector, column, and detector temperatures were kept $60^{\circ}, 100^{\circ}$, and $110^{\circ} \mathrm{C}$, respectively. Methane standard samples $(22.4 \%$, Chemix Specialty Gases and Equipment, Bengaluru, India) were run before and after analysis of the test samples. Based on the area and percentage of the standard gas, the percentage of methane in test sample was calculated using the following formula:

$\mathrm{CH} 4(\%)=(($ standard $\mathrm{CH} 4$ concentration $) \times($ area of test sample))/(area of standard CH4) 


\section{Rumen fermentation characteristics}

On the termination of incubation ( $24 \mathrm{~h}$ ), the buffered rumen fluid was transferred into ice-cold graduated tube to stop further fermentation and $\mathrm{pH}$ was measured (Eutech Instruments, $\mathrm{pH}$ meter Cyberscan) immediately. The samples were, later on, stored at $-20^{\circ} \mathrm{C}$ until further analysis. Stored rumen liquor samples were thawed and used for analyzing ammonia-N [15] by taking $1 \mathrm{~mL}$ of buffered rumen liquor in the outer chamber of the dish, $1 \mathrm{~mL}$ boric acid indicator in the central chamber, and $1 \mathrm{~mL} \mathrm{45 \%}$ potassium carbonate solution to the opposite side of rumen fluid. The content was mixed and titrated against standard sulfuric acid. Total volatile fatty acid (TVFA) in the samples was estimated according to Barnett and Reid [16]. In brief, $1 \mathrm{~mL}$ rumen fluid with an equal volume of buffer (potassium oxalate + oxalic acid) was taken in Markham apparatus, and distillate was collected in a flask placed in ice bath. Few drops of phenolphthalein indicator were added to $100 \mathrm{~mL}$ distillate and then titrated against alkali.

\section{Rumen protozoa enumeration}

Incubation fluid for the enumeration of protozoa was preserved by adding formaldehyde solution $(37-41 \%)$ in $1: 2$. The rumen protozoa were enumerated with the help of counting chamber having a depth of $0.1 \mathrm{~mm}$. The protozoa numbers were calculated according to the study of Kamra et al. [17]. Rumen protozoa based on their morphological characteristics were categorized and were identified to generic level and classified into small spirotrichs mainly entodinimorphs (with an average size of $42 \mu \mathrm{m} \times 23 \mu \mathrm{m}$ ) and large spirotrichs, i.e., diplodinia (average size of $132 \mu \mathrm{m} \times 966 \mu \mathrm{m})$.

\section{Statistical analysis}

Data were analyzed using SPSS [18] version 20.0 software package. The effect of sample and sample with PEG addition on various parameters was analyzed in repeated measures ANOVA. The repeated effect is the number of observations/replicates and incubation. Results were presented as means and standard error of means. The significance was checked by comparing mean values using Tukey's test.

\section{Results}

The nutrient composition and phenol content of the leaf samples known for ethnomedicinal properties are presented in Table- 1 . The CP content of the test phyto-sources ranged between 62 and $198 \mathrm{~g} / \mathrm{Kg}$ dry matter (DM). The CP content in Leucas linifolia was highest (198), while Michelia champaca reported with lowest CP (62). Neutral detergent fiber (NDF) varied $(\mathrm{p}<0.05)$ among the phyto-sources. Litchi chinensis had highest NDF (693), while Citrus grandis had the lowest (295). Similarly, the ADF content also varied $(p<0.05)$ among the samples. The TP content of the phyto-sources varied considerably and reported as high as $211 \mathrm{~g} / \mathrm{kg} \mathrm{DM} \mathrm{(Syzygium} \mathrm{cumini)} \mathrm{and} \mathrm{low} \mathrm{as} 11 \mathrm{~g} / \mathrm{Kg}$ DM (Ficus hispida). Leaves from Terminalia chebula, S. cumini, and Melastoma malabathricum possess highest tannin content ( $>100 \mathrm{~g} / \mathrm{Kg} \mathrm{DM})$; however, the leaves from F. hispida and Alstonia scholaris had negligible tannin content. The CT varied between 0.24 ( $L$. linifolia) and $50.4 \mathrm{~g} / \mathrm{Kg}$ DM (Machilus bombycina). Among the studied samples, S. cumini, M. malabathricum, and T. chebula are high HT-containing sources, while Flacourtia jangomos, Lagerstroemia speciosa,

Table-1: Nutrient content and phenol content of medicinal plant leaves ( $\mathrm{g} / \mathrm{kg} \mathrm{DM})$ from Northeast state, Assam.

\begin{tabular}{|c|c|c|c|c|c|c|c|c|}
\hline Common name & Scientific name & $\mathbf{C P}^{1}$ & NDF $^{1}$ & ADF $^{1}$ & $\mathbf{T P}^{2}$ & $\mathbf{T T}^{\mathbf{2 a}}$ & $\mathbf{C T}^{2 \mathrm{~b}}$ & $\mathbf{H T}^{2 \mathrm{c}}$ \\
\hline Jarul & Lagerstroemia speciosa & $119^{g h}$ & $668^{b}$ & $535^{b}$ & $57.7^{i}$ & $49.8^{h}$ & $1.15^{q}$ & $48.6^{f}$ \\
\hline Panpatti & Christella parasitica (L.) Lev. & $143^{\text {de }}$ & $378^{i}$ & $257^{j}$ & $65.8^{\mathrm{gh}}$ & $50.6^{h}$ & $3.68^{\mathrm{m}}$ & $46.9^{f}$ \\
\hline Droton & Leucas linifolia & $198^{\mathrm{a}}$ & $338^{k}$ & $230^{k}$ & $22.0^{1}$ & $12.5^{k}$ & $0.24^{r}$ & $12.3^{i}$ \\
\hline Hairy fig & Ficus hispida & $115^{\mathrm{h}}$ & $519^{d}$ & $396^{e}$ & $11.0^{\mathrm{n}}$ & $7.13^{\prime}$ & $1.24^{\mathrm{a}}$ & $5.88^{j k}$ \\
\hline Black currant & Antidesma ghaesembilla & $187^{\mathrm{b}}$ & $488^{e}$ & $470^{c}$ & $20.3^{1}$ & $16.9^{j}$ & $10.3^{i}$ & $6.67^{\mathrm{jk}}$ \\
\hline Indian olive & Elaeocarpus floribundus & $163^{c}$ & $384^{\text {hi }}$ & $251^{j}$ & $26.5^{k}$ & $24.0^{i}$ & $4.51^{\prime}$ & $19.5^{h}$ \\
\hline Jamun & Syzygium cumini & $78^{j}$ & $405^{9}$ & $379^{f}$ & $211^{a}$ & $190^{\mathrm{a}}$ & $23.9^{f}$ & $166^{a}$ \\
\hline Burmese grape & Baccaurea ramiflora & $68^{k}$ & $321^{\prime}$ & $259^{j}$ & $94.8^{f}$ & $86.2^{e}$ & $38.8^{\mathrm{d}}$ & $47.5^{f}$ \\
\hline Lichee & Litchi chinensis & $97^{i}$ & $693^{a}$ & $612^{a}$ & $68.6^{g}$ & $61.4^{f}$ & $39.4^{c}$ & $22.0^{\mathrm{h}}$ \\
\hline Rusty kamala & Mallotus ferrugineus & $187^{\mathrm{b}}$ & $450^{f}$ & $384^{\mathrm{ef}}$ & $12.4^{\mathrm{mn}}$ & $9.25^{\prime}$ & $1.24^{\mathrm{a}}$ & $8.00^{j}$ \\
\hline Indian rose chestnut & Mesua ferrea & $78^{j}$ & $560^{c}$ & $416^{d}$ & $64.7^{\mathrm{h}}$ & $57.5^{\mathrm{g}}$ & $30.9^{e}$ & $26.6^{g}$ \\
\hline Singapore rhododendron & Melastoma malabathricum & $131^{f}$ & $364^{j}$ & $258^{j}$ & $124^{c}$ & $118^{c}$ & $9.14^{j}$ & $109^{c}$ \\
\hline Coffee plum & Flacourtia jangomos & $92^{i}$ & $354^{j}$ & $211^{\prime}$ & $121^{d}$ & $97.7^{d}$ & $5.16^{k}$ & $92.5^{d}$ \\
\hline Pomelo & Citrus grandis (L.) osb. & $160^{c}$ & $295^{m}$ & $263^{i j}$ & $14.1^{\mathrm{mn}}$ & $8.52^{\prime}$ & $1.79^{p}$ & $6.72^{\mathrm{jk}}$ \\
\hline Som & Machilus bombycina & $123^{g}$ & $521^{d}$ & $469^{c}$ & $62.7^{\mathrm{h}}$ & $54.4^{g}$ & $50.4^{a}$ & $3.97^{k}$ \\
\hline Chiko & Achras zapota & $69^{k}$ & $462^{f}$ & $337^{g}$ & $108^{e}$ & $96.8^{d}$ & $44.4^{b}$ & $52.3^{e}$ \\
\hline Agarwood & Aquilaria malaccensis & $145^{d}$ & $394^{\text {gh }}$ & $273^{i}$ & $34.6^{j}$ & $26.0^{i}$ & $10.8^{g}$ & $15.2^{i}$ \\
\hline ChebulicMyrobalan & Terminalia chebula & $145^{d}$ & $453^{f}$ & $309^{h}$ & $161^{\mathrm{b}}$ & $154^{\mathrm{b}}$ & $10.5^{\mathrm{h}}$ & $144^{\mathrm{b}}$ \\
\hline Devil tree & Alstonia scholaris & $138^{e}$ & $393^{g h}$ & $211^{\prime}$ & $12.1^{\mathrm{n}}$ & $7.55^{\prime}$ & $1.97^{\circ}$ & $5.58^{\mathrm{jk}}$ \\
\hline Golden champaca & Michelia champaca & $62^{\prime}$ & $492^{e}$ & $413^{d}$ & $15.3^{\mathrm{m}}$ & $9.55^{\mathrm{kl}}$ & $3.39^{n}$ & $6.17^{\mathrm{jk}}$ \\
\hline SEM & & 4.63 & 11.8 & 12.5 & 5.01 & 4.70 & 1.49 & 4.34 \\
\hline p value & & $<0.001$ & $<0.001$ & $<0.001$ & $<0.001$ & $<0.001$ & $<0.001$ & $<0.001$ \\
\hline
\end{tabular}

$\mathrm{CP}=$ Crude Protein, NDF=Neutral detergent fiber, ADF=Acid detergent fiber, $\mathrm{TP}=$ Total phenol, $T \mathrm{~T}=$ Total tannin,

$\mathrm{CT}=$ Condensed tannins, $\mathrm{HT}=$ Hydrolysable tannins. ${ }^{1}$ Each value is the mean of four observations. ${ }^{2}$ Each value is the mean of six observations, a As tannic acid equivalent, b As leucocyanidin equivalent, cDifference of a and b. SEM=Standard error of mean, $\mathrm{DM}=$ Dry matter. Mean value bearing different superscript in a column are significant $(p<0.05)$ 
and Christella parasitica (L.) Lev have medium HT content and F. hispida, Elaeocarpus floribundus, Mallotus ferrugineus, C. grandis (L.) osb., A. scholaris, M. champaca, Aquilaria malaccensis, Antidesma ghaesembilla, and L. linifolia have very low HT content. On the other hand, M. bombycina is a potent source of CT, whereas Achras zapota, L. chinensis, Baccaurea ramiflora, and Mesua ferrea contain good proportion of both CT and HT. A. malaccensis and $A$. ghaesembilla are the low CT-containing sources.

\section{Total gas and methane production}

Data pertaining to the effect of different leaves and PEG addition on total gas and methane production are presented in Table- 2 . Results indicated statistically significant $(\mathrm{p}<0.001)$ variation in total gas production among the studied samples. C. parasitica produced maximum gas ( $33.3 \mathrm{ml} / 200 \mathrm{mg} \mathrm{DM})$ on the incubation, while Lagerstroemia speciosa, M. malabathricum, T. chebula, and L. chinensis, on the other hand, produced very less total gas. The effect of CT-containing sources such as $L$. chinensis and $M$. bombycina on total gas production was comparable (10.0-16.7 ml/200 mg DM) to the HT sources such as T. chebula and S. cumini. However, the phyto-sources having appreciable concentration of both CT and HT (M. ferrea and A. zapota) showed comparatively less impact on gas production than either CT or HT. The adverse effect of tannin on gas production was attenuated with PEG6000 addition, and a significant change $(p<0.001)$ in gas production was noticed. The increase in gas production due to the inclusion of PEG as represented by tannin bioassay (TBA) was highest in M. malabathricum (128) followed by L. speciosa (100) (Table-2). Among the samples, L. chinensis, M. malabathricum, L. speciosa, S. cumini, and T. chebula produced lowest methane (ml/200 $\mathrm{mg} \mathrm{DM})$, while $C$. parasitica, $C$. grandis, L. linifolia, and A. malaccensis produced comparatively more methane. The methane production from the phyto-sources-containing HT was lesser than those with high CT content.

\section{Fermentation characteristics}

Rumen $\mathrm{pH}$ was affected $(\mathrm{p}<0.05)$ with incubation source; however, no notable depression in ruminal $\mathrm{pH}$ was observed below 6.47. Similarly, TVFA production also varied among the samples. Results demonstrated that CT-containing sources such as M. bombycina and $L$. chinensis and promising HT sources such as $T$. chebula and S. cumini have less effect on TVFA production, while a few HT sources such as M. malabathricum and L. speciosa adversely affect TVFA production to the maximum extent (Table-3). PEG6000 addition shows an increase in TVFA production $(p<0.001)$. Tannin from the leaves also affected rumen ammonia-N significantly $(\mathrm{p}<0.001)$. Sources such as T. chebula, A. scholaris, and M. champaca lower ammonia $(\mathrm{p}<0.05)$ production. The adverse impact of tannin on ammonia-N was attenuated with PEG $(\mathrm{p}<0.001)$ addition. Samples rich in HT content (S. cumini, M. malabathricum, and E. floribundus) show highest increase in percentage of ammonia-N as

Table-2: Effect of PEG-6000 addition on in vitro total gas and methane production ( $\mathrm{ml} / 200 \mathrm{mg}$ DM) from medicinal plant leaves.

\begin{tabular}{|c|c|c|c|c|c|c|}
\hline \multirow[t]{2}{*}{ Scientific name } & \multicolumn{2}{|c|}{$\begin{array}{c}\text { Total }{ }^{\#} \text { gas } \\
(\mathrm{ml} / 200 \mathrm{mg} \mathrm{DM}) *\end{array}$} & \multirow[t]{2}{*}{ TBA* } & \multicolumn{2}{|c|}{$\begin{array}{c}\text { Methane } \\
(\mathrm{ml} / 200 \mathrm{mg} \mathrm{DM}) *\end{array}$} & \multirow[t]{2}{*}{$\begin{array}{l}\text { Methane reduction per } \mathrm{ml} \\
\text { of total gas reduction* }\end{array}$} \\
\hline & -PEG & +PEG & & -PEG & +PEG & \\
\hline Lagerstroemia speciosa & $10.4^{i}$ & $20.8^{\mathrm{f}}$ & $100^{\mathrm{ab}}$ & $1.44^{\mathrm{ghi}}$ & $2.96^{\mathrm{hi}}$ & $0.14^{\mathrm{e}}$ \\
\hline Christella parasitica (L.) Lev. & $33.3^{\mathrm{a}}$ & $34.9^{b}$ & $5.00^{f}$ & $5.84^{a}$ & $6.34^{d}$ & $0.30^{\text {cde }}$ \\
\hline Leucas linifolia & $28.2^{\mathrm{b}}$ & $30.5^{d}$ & $8.40^{f}$ & $5.40^{\mathrm{ab}}$ & $6.68^{\mathrm{cd}}$ & $0.65^{a}$ \\
\hline Ficus hispida & $15.9^{g h}$ & $29.4^{d}$ & $84.8^{\mathrm{bc}}$ & $1.78^{\mathrm{ghi}}$ & $4.50^{\mathrm{ef}}$ & $0.20^{\mathrm{e}}$ \\
\hline Antidesma ghaesembilla & $14.0^{\mathrm{h}}$ & $15.3^{\mathrm{h}}$ & $9.60^{f}$ & $1.92^{\mathrm{gh}}$ & $2.28^{\mathrm{hi}}$ & $0.28^{\text {cde }}$ \\
\hline Elaeocarpus floribundus & $17.6^{\mathrm{efg}}$ & $20.6^{f}$ & $18.0^{\text {ef }}$ & $3.06^{\mathrm{ef}}$ & $4.70^{\text {ef }}$ & $0.58^{\mathrm{abc}}$ \\
\hline Syzygium cumini & $16.1^{9}$ & $20.8^{f}$ & $30.4^{\mathrm{ef}}$ & $1.42^{\mathrm{ghi}}$ & $4.10^{\mathrm{fg}}$ & $0.63^{\mathrm{ab}}$ \\
\hline Baccaurea ramiflora & $18.6^{\text {def }}$ & $30.9^{d}$ & $66.8^{\mathrm{cd}}$ & $4.46^{\mathrm{cd}}$ & $7.54^{\mathrm{bc}}$ & $0.25^{e}$ \\
\hline Litchi chinensis & $10.0^{i}$ & $16.1^{\mathrm{g}}$ & $61.6^{\mathrm{cd}}$ & $1.04^{i}$ & $2.12^{i}$ & $0.18^{e}$ \\
\hline Mallotus ferrugineus & $16.0^{\mathrm{g}}$ & $18.2^{\mathrm{g}}$ & $13.8^{\mathrm{ef}}$ & $1.80^{\mathrm{ghi}}$ & $2.72^{\mathrm{hi}}$ & $0.41^{\text {abcde }}$ \\
\hline Mesua ferrea & $19.3^{\text {de }}$ & $34.9^{b}$ & $80.6^{\mathrm{bc}}$ & $3.12^{\mathrm{e}}$ & $8.00^{\mathrm{b}}$ & $0.31^{\text {cde }}$ \\
\hline Melastoma malabathricum & $10.8^{i}$ & $24.2^{\mathrm{e}}$ & $128^{\mathrm{a}}$ & $1.12^{\mathrm{hi}}$ & $3.20^{\mathrm{gh}}$ & $0.16^{\mathrm{e}}$ \\
\hline Flacourtia jangomos & $24.7^{c}$ & $30.4^{d}$ & $23.2^{\mathrm{ef}}$ & $4.88^{\mathrm{bcd}}$ & $7.04^{\mathrm{bcd}}$ & $0.38^{\mathrm{abcde}}$ \\
\hline Citrus grandis (L.) osb. & $27.8^{b}$ & $36.9^{a}$ & $32.6^{\mathrm{ef}}$ & $5.52^{\mathrm{ab}}$ & $10.8 a$ & $0.58^{\mathrm{abc}}$ \\
\hline Machilus bombycina & $16.7^{\mathrm{fg}}$ & $22.0^{f}$ & $32.4^{\text {ef }}$ & $2.12^{g}$ & $5.14^{\mathrm{ef}}$ & $0.58^{\mathrm{abcd}}$ \\
\hline Achras zapota & $20.6^{d}$ & $37.7^{\mathrm{a}}$ & $83.0^{\mathrm{bc}}$ & $2.24^{\mathrm{fg}}$ & $8.08^{b}$ & $0.34^{\text {bcde }}$ \\
\hline Aquilaria malaccensis & $29.4^{\mathrm{b}}$ & $34.2^{c}$ & $16.4^{\mathrm{ef}}$ & $5.24^{\mathrm{abc}}$ & $6.52^{\mathrm{cd}}$ & $0.27^{\mathrm{de}}$ \\
\hline Terminalia chebula & $10.4^{i}$ & $14.4^{\mathrm{h}}$ & $38.6^{\text {de }}$ & $1.82^{\mathrm{ghi}}$ & $2.96^{\mathrm{hi}}$ & $0.28^{\text {cde }}$ \\
\hline Alstonia scholaris & $24.1^{c}$ & $29.4^{d}$ & $22.0^{\text {ef }}$ & $4.24^{d}$ & $6.56^{\mathrm{cd}}$ & $0.44^{\mathrm{abcde}}$ \\
\hline Michelia champaca & $18.3^{\text {ef }}$ & $20.8^{f}$ & $13.6^{\mathrm{ef}}$ & $4.24^{d}$ & $5.18^{e}$ & $0.39^{\mathrm{abcde}}$ \\
\hline SEM & 0.67 & 0.75 & 3.67 & 0.17 & 0.23 & 0.02 \\
\hline $\mathrm{p}$ value & $<0.001$ & $<0.001$ & $<0.001$ & $<0.001$ & $<0.001$ & $<0.001$ \\
\hline
\end{tabular}

$\mathrm{PEG}=$ Polyethylene glycol, TBA=Tannin bioassay, $\mathrm{SEM}=$ Standard error of mean. *Each value is the mean of five

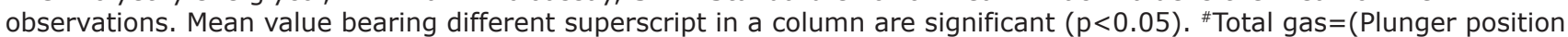
at $24 \mathrm{~h}$-plunger position at $0 \mathrm{~h}=\mathrm{x}$ ml-average of gas produced in blank syringe=Total gas $/ 200 \mathrm{mg}$ of sample, which is converted to the DM content of sample, thus total gas/200 mg DM). DM=Dry matter 
compared to the rest of the samples on PEG inclusion in our study.

\section{Effect on rumen protozoa population}

Total protozoa numbers $\left(\times 10^{5}\right)$ varied $(\mathrm{p}<0.05)$ considerably (0.122-0.262) among the phyto-sources (Table-4). C. parasitica, T. chebula, and M. bombycina exhibited maximum influence on rumen protozoa population. Phyto-sources such as M. malabathricum and $F$. jangomos that contain high HT did not affect rumen protozoa. Similarly, the effect of $M$. ferrea, a good source of both HT and CT, also did not affect the ciliate population. PEG addition to the basal diet increased $(p<0.05)$ the protozoal numbers in comparison of their respective control. In this study, entodinia were found major ciliates (Table-4); however, their numbers also varied $(p<0.05)$ among the studied samples.

\section{Discussion}

Northeast India from where phyto-sources used in this study were collected forms a unique biogeographic province in the world by sharing a part of both Himalayan and Indo-Burmese biodiversity hotspots [10]. The phyto-sources were evaluated in vitro for their anti-methanogenic properties to supplement the knowledge and broaden the list of feed additives which induces positive changes by altering rumen fermentation to achieve significant methane reduction. Among the studied leaves, $C$. parasitica produced maximum in vitro gas, while $L$. speciosa, T. chebula, L. chinensis, and M. malabathricum produced minimum. To ascertain the effect of tannin on gas production, PEG-6000 was used in our study. PEG-6000 neutralizes the effect of tannin by forming tannin-PEG complex and thereby increases carbohydrate digestibility $[19,20]$. In this study, wide variation in gas production was seen among the phyto-sources and the addition of PEG-6000 has shown almost double-fold increase in the gas production, suggesting the influence of tannin on carbohydrates. However, PEG addition to the basal diet always not increased fermentation of the tannins-containing basal diet. This unresponsive mechanism of PEG towards tannins could be attributed to the less bacterial adhesion to the feed because of PEG [21]. Tannins (both CT and HT) interact with carbohydrate by hydrogen bonding and hydrophobic interaction due to its large number of hydroxyl group [22,23]. However, difference in tannin polymer size is one of the main factors affecting its ability to bind to fiber [24], which influences fermentation of tannin-containing phyto-sources. The high tannin content of T. chebula and M. malabathricum produced relatively low gas than $L$. linifolia and A. scholaris which contained comparatively low tannin [25]. These results clearly indicated that tannin level affects the feed fermentation and subsequent gas production. The difference in gas production among the phyto-sources could also be due to the presence of prodelphidin. CT with larger proportion of prodelphidin has high affinity toward fiber as prodelphidin has more hydrogen bonding sites, thus creating a CT-fiber complex which reduces fiber degradation and affects gas production differently [26]. In our study, we have not estimated prodelphidin; however, there presence

Table-3: Effect of medicinal plant leaves and PEG addition on rumen fermentation characteristics.

\begin{tabular}{|c|c|c|c|c|c|c|}
\hline \multirow[t]{2}{*}{ Common name } & \multicolumn{2}{|c|}{$\mathbf{p H} *$} & \multicolumn{2}{|c|}{ TVFA (mmol/dL)* } & \multicolumn{2}{|c|}{ Ammonia nitrogen ( $\mathrm{mg} / \mathrm{dL}) *$} \\
\hline & -PEG & +PEG & -PEG & +PEG & -PEG & +PEG \\
\hline Lagerstroemia speciosa & $7.03^{a b}$ & $7.04^{\mathrm{ab}}$ & $8.50^{f}$ & $9.23^{1}$ & $25.9^{a b}$ & $27.8^{a b}$ \\
\hline Christella parasitica (L.) Lev. & $6.73^{\mathrm{gh}}$ & $6.77^{\mathrm{h}}$ & $11.8^{\mathrm{bc}}$ & $14.6^{\mathrm{ab}}$ & $27.5^{a}$ & $29.6^{a}$ \\
\hline Leucas linifolia & $6.96^{\mathrm{bcd}}$ & $6.97^{\mathrm{abcd}}$ & $12.0^{\mathrm{abc}}$ & $13.4^{\text {de }}$ & $19.8^{\text {defg }}$ & $22.2^{\mathrm{efg}}$ \\
\hline Ficus hispida & $6.47^{i}$ & $6.50^{\mathrm{i}}$ & $10.1^{\text {de }}$ & $11.2^{i j k}$ & $23.3^{b c}$ & $25.9^{\mathrm{bcd}}$ \\
\hline Antidesma ghaesembilla & 6.89 de & $7.02^{\mathrm{abc}}$ & $12.5^{\mathrm{ab}}$ & $13.4^{\text {def }}$ & $21.0^{\text {cde }}$ & $27.8^{\mathrm{ab}}$ \\
\hline Elaeocarpus floribundus & $6.99^{a b c}$ & $7.03^{a b c}$ & $10.1^{\text {de }}$ & $11.7^{\mathrm{hi}}$ & $18.2^{\mathrm{fgh}}$ & $25.0^{\mathrm{cd}}$ \\
\hline Syzygium cumini & $7.05^{\mathrm{a}}$ & $7.06^{a}$ & $10.1^{\text {de }}$ & $10.7^{\mathrm{kj}}$ & $20.3^{\text {defg }}$ & $25.9^{\mathrm{bcd}}$ \\
\hline Baccaurea ramiflora & $6.78^{\mathrm{fg}}$ & $6.88^{\text {efg }}$ & $12.0^{\mathrm{abc}}$ & $13.7^{\mathrm{cd}}$ & $15.6^{h}$ & $19.8^{g}$ \\
\hline Litchi chinensis & $6.92^{\mathrm{cd}}$ & $6.95^{\text {cde }}$ & $12.1^{\mathrm{abc}}$ & $12.7^{\mathrm{fg}}$ & $25.7^{a b}$ & $27.3^{\mathrm{abc}}$ \\
\hline Mallotus ferrugineus & $6.88^{\text {de }}$ & $6.89^{\mathrm{defg}}$ & $13.0^{\mathrm{ab}}$ & $14.9^{a}$ & $21.2^{\text {cde }}$ & $21.5^{\mathrm{fg}}$ \\
\hline Mesua ferrea & $6.90^{\mathrm{de}}$ & $6.92^{\text {de }}$ & $11.8^{\mathrm{bc}}$ & $14.8^{\mathrm{ab}}$ & $21.7^{\mathrm{cd}}$ & $25.0^{\mathrm{cd}}$ \\
\hline Melastoma malabathricum & $7.01^{\mathrm{ab}}$ & $7.04^{\mathrm{ab}}$ & $9.77^{e}$ & $10.5^{k}$ & $17.7^{\mathrm{gh}}$ & $24.0^{\text {def }}$ \\
\hline Flacourtia jangomos & $6.74^{\mathrm{gh}}$ & $6.75^{\mathrm{h}}$ & $12.1^{\mathrm{abc}}$ & $13.5^{d}$ & $19.8^{\text {defg }}$ & $20.8^{g}$ \\
\hline Citrus grandis (L.) osb. & $6.89^{\text {de }}$ & $6.91^{\text {def }}$ & $11.1^{\mathrm{cd}}$ & $12.1^{\mathrm{gh}}$ & $26.1^{\mathrm{a}}$ & $27.8^{\mathrm{ab}}$ \\
\hline Machilus bombycina & $6.95^{\mathrm{bcd}}$ & $6.96^{\text {bcde }}$ & $13.0^{\mathrm{ab}}$ & $14.2^{\mathrm{bc}}$ & $22.4^{\mathrm{cd}}$ & $24.0^{\text {def }}$ \\
\hline Achras zapota & $6.75^{\mathrm{fgh}}$ & $6.76^{\mathrm{h}}$ & $10.1^{\text {de }}$ & $10.7^{\mathrm{kj}}$ & $20.5^{\text {def }}$ & $24.3^{\text {de }}$ \\
\hline Aquilaria malaccensis & $6.68^{\mathrm{h}}$ & $6.83^{\mathrm{fgh}}$ & $12.1^{\mathrm{abc}}$ & $12.8^{\mathrm{ef}}$ & $18.7^{\text {efg }}$ & $21.5^{\mathrm{fg}}$ \\
\hline Terminalia chebula & $6.83^{\mathrm{ef}}$ & $6.90^{\text {defg }}$ & $13.1^{a}$ & $13.6^{\mathrm{cd}}$ & $7.70^{\mathrm{j}}$ & $8.38^{i}$ \\
\hline Alstonia scholaris & $6.91^{\text {cde }}$ & $7.06^{a}$ & $10.6^{\text {de }}$ & $12.0^{\mathrm{h}}$ & $6.53^{j}$ & $7.47^{i}$ \\
\hline Michelia champaca & $6.75^{\mathrm{fgh}}$ & $6.81^{\mathrm{gh}}$ & $10.1^{\text {de }}$ & $11.2^{\mathrm{ij}}$ & $10.7^{i}$ & $11.9^{\mathrm{h}}$ \\
\hline SEM & 0.01 & 0.01 & 0.12 & 0.15 & 0.53 & 0.57 \\
\hline$p$ value & $<0.001$ & $<0.001$ & $<0.001$ & $<0.001$ & $<0.001$ & $<0.001$ \\
\hline
\end{tabular}

$\mathrm{PEG}=$ Polyethylene glycol, TVFA=Total volatile fatty acids, SEM=Standard error of mean. *Each value is the mean of six observations. Mean value bearing different superscript in a column are significant $(p<0.05)$ 
Table-4: Effect of medicinal plant leaves on rumen protozoal population.

\begin{tabular}{|c|c|c|c|c|c|c|c|}
\hline \multirow[t]{2}{*}{ Common name } & \multicolumn{2}{|c|}{ Entodina $\left(\times 10^{5}\right) *$} & \multicolumn{2}{|c|}{ Holotricha $\left(\times 10^{5}\right) *$} & \multicolumn{2}{|c|}{$\begin{array}{c}\text { Total } \\
\text { protozoa }\left(\times 10^{5}\right)^{*}\end{array}$} & \multirow[t]{2}{*}{$\begin{array}{c}\% \\
\text { increase* }\end{array}$} \\
\hline & -PEG & +PEG & -PEG & +PEG & -PEG & +PEG & \\
\hline Lagerstroemia speciosa & $0.176^{\text {def }}$ & $0.185^{\text {cdefg }}$ & $0.003^{\mathrm{cd}}$ & $0.006^{\mathrm{cd}}$ & $0.178^{\mathrm{cd}}$ & $0.191^{\text {efghij }}$ & $6.84^{\text {cde }}$ \\
\hline Christella parasitica (L.) Lev. & $0.125^{i}$ & $0.157^{\text {ghij }}$ & $0.003^{\mathrm{cd}}$ & $0.017^{\mathrm{ab}}$ & $0.128^{\mathrm{fg}}$ & $0.174^{\text {hijk }}$ & $37.5^{\mathrm{ab}}$ \\
\hline Leucas linifolia & $0.183^{\text {cdef }}$ & $0.220^{c}$ & $0.003^{\mathrm{cd}}$ & $0.008^{\mathrm{bcd}}$ & $0.187^{\mathrm{bc}}$ & $0.228^{\mathrm{cd}}$ & $23.0^{\mathrm{bcd}}$ \\
\hline Ficus hispida & $0.192^{\mathrm{bcd}}$ & $0.212^{\mathrm{cd}}$ & $0.001^{d}$ & $0.002^{c d}$ & $0.193^{b c}$ & $0.214^{\text {cdef }}$ & $10.8^{\text {bcde }}$ \\
\hline Antidesma ghaesembilla & $0.173^{\text {def }}$ & $0.172^{\text {efghi }}$ & $0.001^{d}$ & $0.004^{c d}$ & $0.173^{\mathrm{cd}}$ & $0.175^{\text {ghijk }}$ & $1.44^{\mathrm{de}}$ \\
\hline Elaeocarpus floribundus & $0.206^{b c}$ & $0.180^{\text {defghi }}$ & $0.002^{\mathrm{cd}}$ & $0.001^{d}$ & $0.208^{b}$ & $0.181^{\text {fghijk }}$ & $0.00^{\mathrm{e}}$ \\
\hline Syzygium cumini & $0.160^{\text {efgh }}$ & $0.206^{\text {cde }}$ & $0.004^{\mathrm{bcd}}$ & $0.006^{\mathrm{cd}}$ & $0.165^{\text {cde }}$ & $0.211^{\text {cdefg }}$ & $31.3^{\mathrm{bc}}$ \\
\hline Baccaurea ramiflora & $0.165^{\text {defg }}$ & $0.179^{\text {defghi }}$ & $0.001^{d}$ & $0.002^{\mathrm{cd}}$ & $0.166^{\text {cde }}$ & $0.181^{\text {fghijk }}$ & $10.4^{\text {bcde }}$ \\
\hline Litchi chinensis & $0.184^{\text {cde }}$ & $0.207^{\text {cde }}$ & $0.002^{\mathrm{cd}}$ & $0.003^{c d}$ & $0.186^{b c}$ & $0.211^{\text {cdefgh }}$ & $13.5^{\text {bcde }}$ \\
\hline Mallotus ferrugineus & $0.191^{\mathrm{bcd}}$ & $0.197^{\text {cdef }}$ & $0.000^{d}$ & $0.001^{c d}$ & $0.192^{\mathrm{bc}}$ & $0.198^{\text {defghi }}$ & $3.70^{\text {cde }}$ \\
\hline Mesua ferrea & $0.210^{\mathrm{bc}}$ & $0.211^{\mathrm{cd}}$ & $0.000^{d}$ & $0.007^{\mathrm{bcd}}$ & $0.210^{\mathrm{b}}$ & $0.219^{\text {cde }}$ & $4.26^{\text {cde }}$ \\
\hline Melastoma malabathricum & $0.213^{b}$ & $0.211^{\mathrm{cd}}$ & $0.001^{d}$ & $0.001^{\mathrm{cd}}$ & $0.214^{b}$ & $0.242^{c}$ & $13.1^{\text {bcde }}$ \\
\hline Flacourtia jangomos & $0.251^{a}$ & $0.279^{b}$ & $0.000^{d}$ & $0.001^{d}$ & $0.251^{a}$ & $0.280^{\mathrm{b}}$ & $11.7^{\text {bcde }}$ \\
\hline Citrus grandis (L.) osb. & $0.260^{\mathrm{a}}$ & $0.429^{a}$ & $0.001^{\mathrm{cd}}$ & $0.000^{d}$ & $0.262^{a}$ & $0.429^{a}$ & $64.0^{a}$ \\
\hline Machilus bombycina & $0.132^{\mathrm{hi}}$ & $0.131^{\mathrm{j}}$ & $0.007^{\mathrm{bc}}$ & $0.017^{\mathrm{ab}}$ & $0.139^{\mathrm{efg}}$ & $0.148^{\mathrm{k}}$ & $7.88^{\text {cde }}$ \\
\hline Achras zapota & $0.139^{g h i}$ & $0.147^{\text {hij }}$ & $0.017^{a}$ & $0.026^{a}$ & $0.156^{\text {def }}$ & $0.172^{\mathrm{ijk}}$ & $10.5^{\text {bcde }}$ \\
\hline Aquilaria malaccensis & $0.155^{\mathrm{fgh}}$ & $0.162^{\text {fghij }}$ & $0.001^{\mathrm{cd}}$ & $0.001^{c d}$ & $0.156^{\text {def }}$ & $0.163^{\mathrm{ijk}}$ & $4.68^{\text {cde }}$ \\
\hline Terminalia chebula & $0.120^{\mathrm{i}}$ & $0.150^{\text {ghij }}$ & $0.001^{c d}$ & $0.004^{c d}$ & $0.122^{\mathrm{g}}$ & $0.154^{\mathrm{jk}}$ & $26.7^{\mathrm{bcd}}$ \\
\hline Alstonia scholaris & $0.141^{\mathrm{ghi}}$ & $0.145^{\mathrm{ij}}$ & $0.010^{\mathrm{b}}$ & $0.011^{b c}$ & $0.151^{\text {defg }}$ & $0.155^{\mathrm{jk}}$ & $3.02^{\text {cde }}$ \\
\hline Michelia champaca & $0.172^{\text {def }}$ & $0.183^{\text {defgh }}$ & $0.001^{d}$ & $0.001^{d}$ & $0.172^{\mathrm{cd}}$ & $0.184^{\text {efghijk }}$ & $6.76^{\text {cde }}$ \\
\hline SEM & 0.004 & 0.002 & 0.000 & 0.001 & 0.002 & 0.006 & 1.96 \\
\hline$p$ value & $<0.001$ & $<.001$ & $<0.001$ & $<0.001$ & $<0.001$ & $<0.001$ & $<0.001$ \\
\hline
\end{tabular}

$\mathrm{PEG}=$ Polyethylene glycol, SEM=Standard error of mean. *Each value is the mean of five observations; Mean value bearing different superscript in a column are significant $(p<0.05)$. A percentage increase represents increase with $P E G$ addition compared to without PEG. Spirotrichs (mainly diplodinia with an average size of $132 \mu \mathrm{m} \times 66 \mu \mathrm{m}$ ). Spirotrichs not identified to generic level were classified into small spirotrichs (mainly entodina with an average size $42 \mu \mathrm{m} \times 23 \mu \mathrm{m}$ ) and large spirotrichs (mainly diplodinia with an average size of $132 \mu \mathrm{m} \times 66 \mu \mathrm{m}$ )

in tannin-containing phyto-sources might be having some effect on the gas production. Apart from the tannin content, non-starch polysaccharide (NSP) [27], fiber fraction, and lignin content also play important role in influencing gas production [28]. Karabulut et al. [29], in an in vitro study, also reported a significant variation in gas production in different legume hays which is attributed to the variable fiber fractions (NDF and ADF). Variation in cell wall content (i.e., NDF and ADF) among different phyto-sources as observed in this study might reduce the availability of rapidly fermented carbohydrates and thereby suppress the microbial activity, resulting in lower gas production. However, it was also found that, with the same carbohydrate content, different gas values can be recorded due to difference in microbial protein synthesis [28]. Studies have also shown that difference in gas production was recorded despite similar tannin and NSP concentration in a study carried out on two Leucaena species (L. leucocephala and L. pallida) in vitro [27]. They stated that this difference could be due to difference in tannin chemistry (molecular size and monomer composition). The different species of phyto-source also determine the ability of fiber to complex with polyphenols, thereby affecting the gas production as observed in a study carried out using 17 Zimbabwean browse species [30]. Hence, the effect of phyto-sources on gas production is an influence of many factors such as its variable tannin, NDF, and ADF content. Furthermore, the variation in tannin polymer size, lignin, NSP, and prodelphidin content which were not estimated in this study might have influenced the gas production. Thus, phyto-sources such as $S$. cumini even with quite large tannin content could not produce less gas as expected.

The effect of tannin-containing phyto-sources on methane production depends largely on fermentation of these phyto-sources, bacteriocidal and bacteriostatic effects of tannin on rumen microbes, and rumen enzymes [25]. The variable tannin biological properties depend on tannin chemical structure and degree of polymerization [31]. Results from our studies revealed variable efficacy of different phyto-sources for methane reduction. This could be attributed to the variable tannin content and tannin structural composition [24] among the studied sources, even though the structural analysis was not carried out in our study. Contrary to high tannin sources such as S. cumini and T. chebula, low methane production was observed in M. malabathricum and $L$. chinensis. This could be due to the presence of other secondary metabolites which might be having additive effect with tannin on methane reduction. For instance, the presence of flavonoids [32], alkaloids, and saponin glycosides [33] which has antimicrobial properties [34,35] in L. chinensis might be responsible for reducing methane along with tannin. Nevertheless, methanogens were not quantified in our study, but it is well-established fact that tannin inhibits methanogenesis directly through methanogen reduction or indirectly through antiprotozoal action, 
thereby restricting $\mathrm{H}_{2}$ transfer to methanogen [8]. The reduction in methane production by $S$. cumini, $T$. chebula, and L. chinensis was supported by a reduced protozoa numbers justifying the indirect effect of tannin on protozoa leading to less methane production. Furthermore, tannins often exhibit a reduction in degradation of nutrients and alter the site of degradation post-ruminally. The study of Hariadi et al. [36] strengthened our results, who reported that tannin content had negative correlation with methane production. Our results demonstrated that $C$. parasitica (L.) and F. jangomos in spite of their high tannin content produced comparatively more methane which could be due to the heterogeneous nature of tannin and a variable degree of polymerization that exerts different biological activities. However, methane production was low from the phyto-sources such as $S$. cumini and T. chebua-containing high HT. Jayanegara et al. [37] also reported that HT has greater ability to reduce methane than $\mathrm{CT}$. Addition of PEG in this study attenuated the adverse impact of tannin on methane production than their corresponding control. Among the studied phyto-sources, L. linifolia (0.65), S. cumini (0.63), E. flribundus (0.58), M. bombycina (0.58), and $C$. grandis $(\mathrm{L}$.) $(0.58)$ were more effective in reducing methane $(\mathrm{ml} / \mathrm{ml}$ of reduction in total gas). The screening of the promising sources for further in vivo evaluation in ruminants before recommending the inclusion level is a big challenge and the criteria should be the reduction in methane per $\mathrm{ml}$ of reduction in total gas production [6]. The sources which have the highest ratio in methane reduction with per unit of gas reduction should be selected for optimizing their level of inclusion in diet and for in vivo evaluation.

The effect of tannins from different sources on rumen $\mathrm{pH}$ remained inconsistent. However, in our study, a significant impact of tannin on $\mathrm{pH}$ was observed which could be attributed to the different soluble carbohydrate content of studied phyto-sources. Previous studies [38,39] also reported that soluble carbohydrate has a well-defined adverse impact on rumen $\mathrm{pH}$. Dschaak et al. [40] studied the effect of CT supplementation on rumen fermentation and found no significant effect of tannin on rumen $\mathrm{pH}$. He suggested the significance of particle size of the diet and its distribution apart from soluble carbohydrate content role in influencing rumen $\mathrm{pH}$.

The adverse impact of high tannin content on feed fermentation is well known where it affects the degradation of fiber in rumen and hence the nutrient availability. There is a wide variation observed in TVFA production from $200 \mathrm{mg}$ of substrate. These results are similar to the study carried out by Bhatta et al. [6,8], where numerous tropical phyto-sources were tested for its methane reduction potential. Phytosources such as M. malabathricum and L. speciosa having high tannin content show significant effect on gas production as well as TVFA. The significant decrease in TVFA production from the phyto-sources appeared an aggregate result of high structural carbohydrate and tannin content. The TVFA which is produced due to the fermentation of feedstuff is mainly constituted by acetate and butyrate, with propionate yielding gas only due to buffering of the acid [41]. The high fiber as well as tannin content or both decreased the fermentability of phyto-sources as evidenced from decreased volatile fatty acid production [25]. However, feeds that produce high amounts of propionate yield lower gas volumes [42]. The presence of non-fiber carbohydrate also contributes to more propionate production [41]. The higher proportion of propionate production leads to a weaker correlation between gas and VFA production [41]. Increase in propionate production is a competitive pathway for methane and thus lowers methane production [43]. In case of $T$. chebula, decrease in methane is evident, so it can be assumed that propionate is increased here. Apart from that, there is a decrease in protozoa population recorded in $T$. chebula. These rumen protozoa ingest starch granules and decrease starch degradation [44]. Hence, in T. chebula, more starch degradation occurs and thus contributes to increases the TVFA content. Similar effect was recorded by Santra and Karim [44] where defaunation effect shows increase in TVFA production. The addition of PEG shows improvement in TVFA production as compared to their corresponding control, indicating the impact of tannin on TVFA production. The different abilities of tannin's phenolic group to bind with PEG as reported earlier [20] could be the reason for variable increase in TVFA production.

Tanniniferous phyto-sources have a most pronounced effect on ammonia-N [45], and it was confirmed in the present study too where PEG addition improved the ammonia- $\mathrm{N}$ concentration in test treatments as compared to the respective control. Tannins have high affinity toward protein and form cross-linkages and therefore decrease the protein degradation in rumen and increase the post-ruminal utilization. Bhatta et al. [46], in an in vitro study, reported the alteration in nitrogen excretion more from urinary- $\mathrm{N}$ to fecal nitrogen and observed an overall improvement in nitrogen utilization due to post-ruminal degradation. Our study recorded similar trend with Bhatta et al. [6], in ammonia-N with $200 \mathrm{mg}$ of incubated sample proving the effect of tannin in reducing ruminal ammonia-N production. The phyto-sources which have both $\mathrm{CT}$ and HT content in them such as A. zapota, M. feea, B. ramiflora, A. ghaesembilla, and A. malaccensis produce more variation in ammonia-N. This huge variation in CT- and HT-containing phyto-sources is due to partially reversible nature of protein-HT complex [6] as rumen bacteria can dissociate protein-HT complex easily than protein-CT complexes. On the other hand, samples which are having more HT content such as S. cumini, L. speciosa, and C. parasitica (L.) Lev have ammonia-N concentration in a narrow range of $(20.3-27.5 \mathrm{mg} / \mathrm{dl})$. However, an interesting result is 
observed in case of $T$. chebula and S. cumini which are both a rich source of HT. The former one produced very low ammonia-N as compared to the later. This low concentration of ammonia-N in T. chebula compared to $S$. cumini could be due to less number of protozoa in the former. This leads to decrease in bacterial protein breakdown and eventually feed protein degradability [47]. The low protozoa number of T. chebula was also observed in Bhatta et al. [8] study. Deaville et al. [48], in a study, reported that the binding strength of tannin-protein complex depends on the polyol core of the HT and also stated that the loss of conformational freedom in tannin structure weakens its bond to protein. Since in this study exact structural elucidation is not done, we cannot assure this, but the difference in the binding ability of HT to protein could also be the reason behind wide variations in ammonia-N content as weakly bound proteins will be easy to degrade and resulting in more ammonia-N than the tightly bound ones. The PEG-tannin interaction showed an increase in ammonia-N production up to $35 \%$ in few of the HT sources, which possibly was due to more hydroxyl $(\mathrm{OH})$ group in HT than CT sources. González et al. [49] had carried out a study where they found that chestnut tannins were more effective in protecting soybean meal than quebracho tannins from in vitro bacterial degradation because of the presence of more $\mathrm{OH}$ group than quebracho tannin as $\mathrm{OH}$ group forms hydrogen bond with $\mathrm{NH}_{2}, \mathrm{SH}$, $\mathrm{OH}$, and $\mathrm{CO}$ groups of proteins.

Protozoa are well known for the interspecies hydrogen transfer to the archaea which use it for the reduction of $\mathrm{CO}_{2}$ into methane [47]. The PEG addition showed an increase in the total protozoa; however, the increase in the total protozoa was not similar across the studied phyto-sources. This variation in protozoal action could be attributed to the variable efficiency of PEG to bind tannin from different sources as well as the structural variation of tannin. In our study, entodinomorphs were major ciliates while holotrichs represented the small fraction of total protozoa irrespective of the phyto-sources. Bhatta et al. [8] also reported that holotrichs were not major group of the rumen protozoa. Previous studies [50] on HT have shown a significant effect on entodina and total protozoa population. In agreement to their results, we have observed a significant effect of S. cumini and T. chebula which are promising HT source on rumen protozoa whose effect is counteracted by PEG addition showing an increase in total protozoa population up to $31.4 \%$. While in M. malabathricum, which is a good tannin source, addition of PEG had increase protozoa population only by $13.1 \%$. A study carried out by Mamat et al. [51] on M. malabathricum reported the presence of other secondary metabolites such as saponins and flavonoids. Both flavonoid $[52,53]$ and saponins are known to reduce ciliate population $[54,55]$. Since PEG6000 is a tannin binder [19], it complexes only with tannin, leaving the flavonoids and saponin [51]. Thus, the expected increase in protozoa population was not observed. Similarly, F. jangomos and L. speciosa have shown to impact rumen protozoa, but PEG addition has shown very minimal effect on protozoa reversal to its equilibrium showing the presence of other secondary metabolites like saponin which is known for having negative effect on rumen protozoa count.

\section{Conclusion}

From this study, it can be concluded that S. cumini, M. malabathricum, and T. chebula arehighHT-containing sources, while F. jangomos, L. speciosa, and C. parasitica (L.) Lev are medium HT-containing sources. On the other hand, M. bombycina is a potent source of CT, whereas A. zapota, L. chinensis, B. ramiflora, and $M$. ferrea contain good proportion of both CT and HT. Among these phyto-sources, L. chinensis, M. malabathricum, L. speciosa, S. cumini, and T. chebula are having potent methane suppressing properties as evidenced by lowest methane production when incubated for $24 \mathrm{~h}$ as compared to the rest of the samples. Further studies on determination of optimum dose of the above samples are required which can be used to carry out in vivo studies so as to establish them as methane-suppressant phyto-source in the ruminant diets.

\section{Authors' Contributions}

$\mathrm{RB}$ and PKM planned and designed the study. The experiment was conducted by LB. APK, AD, $\mathrm{PKM}$, and RB gave necessary inputs during the experimental phases and also allowed the use of instruments for smooth running of the experiment. RB and PKM carried out interpretation of results. LB and PKM participated in data analysis and also involved in the preparation of a draft of the manuscript. All authors read and approved the final manuscript.

\section{Acknowledgments}

The financial support from the Indian Council of Agricultural Research, New Delhi, for carrying this work under the outreach project on "Estimation of methane emission under different feeding systems and development of mitigation strategies" is duly acknowledged. The authors are thankful to the Director, National Institute of Animal Nutrition and Physiology for extending all the support to undertake this work. First author of the manuscript is also thankful to Jain University Faculty of Sciences, where lead author is registered for doctoral program, for their constant support and guidance.

\section{Competing Interests}

The authors declare that they have no competing interests.

\section{References}

1. Johnson, K.A. and Johnson, D.E. (1995) Methane emissions from cattle. J. Anim. Sci., 73(8): 2483-2492.

2. Guan, H., Wittenberg, K.M., Ominski, K.H. and Krause, D.O. (2006) Efficacy of ionophores in cattle diets for mitigation of enteric methane. J. Anim. Sci., 84(7): 1896-1906. 
3. Malik, P.K., Bhatta, R., Soren, N.M., Sejian, V., Mech, A., Prasad, K.S. and Prasad, C.S. (2015) Feed Based Approaches in Enteric Methane Amelioration. Livestock Production and Climate Change. CAB International, Wallingford. p336-359.

4. Malik, P.K., Kolte, A.P., Dhali, A., Sejian, V., Thirumalaisamy, G., Gupta, R. and Bhatta, R. (2016) GHG emissions from Livestock: Challenges and ameliorative measures to counter adversity. In Greenhouse GasesSelected Case Studies. InTech, Janeza Trdine 9, 51000 Rijeka, Croatia.

5. Guo, Y.Q., Liu, J.X., Lu, Y., Zhu, W.Y., Denman, S.E. and McSweeney, C.S. (2008) Effect of tea saponin on methanogenesis, microbial community structure and expression of $m c r A$ gene, in cultures of rumen micro-organisms. Lett. Appl. Microbiol., 47(5): 421-426.

6. Bhatta, R., Saravanan, M., Baruah, L. and Sampath, K.T. (2012) Nutrient content, in vitro ruminal fermentation characteristics and methane reduction potential of tropical tannin-containing leaves. J. Sci. Food Agric., 92(15): 2929-2935.

7. Patra, A.K. and Yu, Z. (2012) Effects of essential oils on methane production and fermentation by, and abundance and diversity of, rumen microbial populations. Appl. Environ. Microbiol., 78(12): 4271-4280.

8. Bhatta, R., Baruah, L., Saravanan, M., Suresh, K.P. and Sampath, K.T. (2013) Effect of medicinal and aromatic plants on rumen fermentation, protozoa population and methanogenesis in vitro. J. Anim. Physiol. Anim. Nutr., 97(3): 446-456.

9. Pal, K., Patra, A.K. and Sahoo, A. (2015) Evaluation of feeds from tropical origin for in vitro methane production potential and rumen fermentation in vitro. Spanian J. Agric. Res., 13(3): 608.

10. Chatterjee, S., Saikia, A., Dutta, P., Ghosh, D., Pangging, G. and Goswami, A.K. (2006) Biodiversity Significance of North East India. WWF India, Delhi, India.

11. AOAC. (1995) Official Methods of Analysis. $16^{\text {th }}$ ed. Association of Official Analytical Chemists, Washington, DC.

12. Van Soest, P.V., Robertson, J.B. and Lewis, B.A. (1991) Methods for dietary fiber, neutral detergent fiber, and nonstarch polysaccharides in relation to animal nutrition. $J$. Dairy Sci., 74(10): 3583-3597.

13. Makkar, H.P. (2003) Quantification of tannins in tree and shrub foliage: A laboratory manual. Springer Science and Business Media, New York.

14. Menke, K.H., Raab, L., Salewski, A., Steingass, H., Fritz, D. and Schneider, W. (1979) The estimation of the digestibility and metabolizable energy content of ruminant feeding stuffs from the gas production when they are incubated with rumen liquor in vitro. J. Agric. Sci., 93(01): 217-222.

15. Conway, E.J. (1957) Micro Diffusion Analysis and Volumetric Error. $4^{\text {th }}$ ed. Crossby Lockwood and Son, London, England.

16. Barnett, A.J.G. and Reid, R.L. (1957) Studies on the production of volatile fatty acids from grass and rumen liquor in an artificial rumen 1 . Volatile fatty acids production from fresh grasses. J. Agric. Sci., 48: 315-321.

17. Kamra, D.N., Sawal, R.K., Pathak, N.N., Kewalramani, N. and Agarwal, N. (1991) Diurnal variation in ciliate protozoa in the rumen of blackbuck (Antilope cervicapra) fed green forage. Lett. Appl. Microbiol., 13(3): 165-167.

18. IBM SPSS. (2011) Statistics for Windows, Version 20.0. IBM Corp, Armonk, New York.

19. Makkar, H.P.S., Blümmel, M. and Becker, K. (1995) Formation of complexes between polyvinyl pyrrolidones or polyethylene glycols and tannins, and their implication in gas production and true digestibility in in vitro techniques. Br. J. Nutr., 73(6): 897-913.

20. Silanikove, N., Perevolotsky, A. and Provenza, F.D. (2001)
Use of tannin-binding chemicals to assay for tannins and their negative post-ingestive effects in ruminants. Anim. Feed Sci. Technol., 91(1-2): 69-81.

21. Besharati, M. and Taghizadeh, A. (2011) Effect of tannin-binding agents (polyethylene glycol and polyvinylpyrrolidone) supplementation on in vitro gas production kinetics of some grape yield byproducts. ISRN Vet. Sci., 2011: 780540 .

22. Haslam, E. (1989). Plant polyphenols: Vegetable tannins revisited. In: Chemistry and Pharmacology of Natural Products. Cambridge University Press, Cambridge.

23. Tang, H.R., Covington, A.D. and Hancock, R.A. (2003). Structure-activity relationships in the hydrophobic interactions of polyphenols with cellulose and collagen. Biopolymers, 70(3): 403-413.

24. Hatew, B., Stringano, E., Mueller-Harvey, I., Hendriks, W.H., Carbonero, C.H., Smith, L.M.J. and Pellikaan, W.F. (2016) Impact of variation in structure of condensed tannins from sainfoin (Onobrychis viciifolia) on in vitro ruminal methane production and fermentation characteristics. J. Anim. Physiol. Anim. Nutr., 100(2): 348-360.

25. Gemeda, B.S. and Hassen, A. (2015) Effect of tannin and species variation on in vitro digestibility, gas, and methane production of tropical browse plants. Asian Australas. J. Anim. Sci., 28(2): 188-199.

26. Huyen, N.T., Fryganas, C., Uittenbogaard, G., MuellerHarvey, I., Verstegen, M.W.A., Hendriks, W.H. and Pellikaan, W.F. (2016) Structural features of condensed tannins affect in vitro ruminal methane production and fermentation characteristics. J. Agric. Sci., 154(8): 1474-1487.

27. Barahona, R., Lascano, C.E., Narvaez, N., Owen, E., Morris, P. and Theodorou, M.K. (2003) In vitro degradability of mature and immature leaves of tropical forage legumes differing in condensed tannin and non-starch polysaccharide content and composition. J. Sci. Food Agric., 83(12): 1256-1266.

28. Nsahlai, I.V., Siaw, D.E.K.A. and Osuji, P.O. (1994) The relationships between gas production and chemical composition of 23 browses of the genus Sesbania. J. Sci. Food Agric., 65(1): 13-20.

29. Karabulut, A., Canbolat, O., Ozkan, C.O. and Kamalak, A. (2007) Determination of nutritive value of citrus tree leaves for sheep using in vitro gas production technique. Asian Australas. J. Anim. Sci., 20(4): 529.

30. Ndlovu, L.R. and Nherera, F.V. (1997) Chemical composition and relationship to in vitro gas production of Zimbabwean browsable indigenous tree species. Anim. Feed. Sci. Technol., 69(1-3): 121-129.

31. Patra, A.K. and Saxena, J. (2011) Exploitation of dietary tannins to improve rumen metabolism and ruminant nutrition. J. Sci. Food. Agric., 91(1): 24-37.

32. Wen, L., Wu, D., Jiang, Y., Prasad, K.N., Lin, S., Jiang, G., He, J., Zhao, M., Luo, W. and Yang, B. (2014) Identification of flavonoids in litchi (Litchi chinensis Sonn.) leaf and evaluation of anticancer activities. J. Funct. Foods, 6: 555-563.

33. Shukla, R.K., Painuly, D., Porval, A. and Shukla, A. (2014) Proximate analysis, nutritional value, phytochemical evaluation, and biological activity of Litchi chinensis Sonn. leaves. J. Herbs Spices Med. Plants, 20(2): 196-208.

34. Patra, A.K. and Saxena, J. (2010) A new perspective on the use of plant secondary metabolites to inhibit methanogenesis in the rumen. Photochemistry, 71(11-12): 1198-1222.

35. dos Santos, E.T., Pereira, M.L.A., da Silva, C.F.P., SouzaNeta, L.C., Geris, R., Martins, D., Santana, A.E.G., Barbosa, L.C.A., Silva, H.G.O., Freitas, G.C. and Figueiredo, M.P. (2013) Antibacterial activity of the alkaloid-enriched extract from Prosopis juliflora pods and its influence on in vitro ruminal digestion. Int. J. Mol. Sci., 14(4): 8496-8516.

36. Hariadi, B.T. and Santoso, B. (2010) Evaluation of tropical plants containing tannin on in vitro methanogenesis and fermentation parameters using rumen fluid. J. Sci. Food Agric., 90(3): 456-461. 
37. Jayanegara, A., Goel, G., Makkar, H.P.S. and Becker, K. (2010) Reduction in methane emissions from ruminants by plant secondary metabolites: Effects of polyphenols and saponins. Sustainable Improvement of Animal Production and Health. Food and Agriculture Organization of the United Nations (FAO), Rome, Italy. p151-157.

38. Yang, W.Z., Beauchemin, K.A and Rode, L.M. (2001) Effects of grain processing, forage to concentrate ratio, and forage particle size on rumen $\mathrm{pH}$ and digestion by dairy cows. J. Dairy Sci., 84(10): 2203-2216.

39. Krause, K.M., Combs, D.K. and Beauchemin, K.A. (2002) Effects of forage particle size and grain fermentability in midlactation cows. II. Ruminal $\mathrm{pH}$ and chewing activity. $J$. Dairy Sci., 85: 1947-1957.

40. Dschaak, C.M., Williams, C.M., Holt, M.S., Eun, J.S., Young, A.J. and Min, B.R. (2011) Effects of supplementing condensed tannin extract on intake, digestion, ruminal fermentation, and milk production of lactating dairy cows. $J$. Dairy Sci., 94(5): 2508-2519.

41. Getachew, G., Robinson, P.H., De Peters, E.J. and Taylor, S.J. (2004) Relationships between chemical composition, dry matter degradation and in vitro gas production of several ruminant feeds. Anim. Feed Sci. Technol., 111(1-4): 57-71.

42. Beuvink, J.M.W. and Spoelstra, S.F. (1992) Interactions between substrate, fermentation end products, buffering systems and gas production upon fermentation of different carbohydrates by mixed rumen microorganisms in vitro. Appl. Microbiol. Biotechnol., 37: 505-509.

43. Moss, A.R., Jouany, J.P. and Newbold, J. (2000) Methane production by ruminants: Its contribution to global warming. Ann. Zootech., 49: 231-253.

44. Santra, A. and Karim, S.A. (2002) Influence of ciliate protozoa on biochemical changes and hydrolytic enzyme profile in the rumen ecosystem. J. Appl. Microbiol., 92(5): 801-811.

45. Makkar, H.P.S., Francis, G. and Becker, K. (2007) Bioactivity of phytochemicals in some lesser-known plants and their effects and potential applications in livestock and aquaculture production systems. Animal, 1: 1371-1391.

46. Bhatta, R., Uyeno, Y., Tajima, K., Takenaka, A., Yabumoto, Y., Nonaka, I., Enishi, O. and Kurihara, M. (2009) Difference in the nature of tannins on in vitro ruminal methane and volatile fatty acid production and on methanogenic archaea and protozoal populations. J. Dairy Sci., 92(11): 5512-5522.

47. Newbold, C.J., de la Fuente, G., Belanche, A., RamosMorales, E. and McEwan, N.R. (2015) The role of ciliate protozoa in the rumen. Front. Microbiol., 6: 1313.

48. Deaville, E.R., Green, R.J., Mueller-Harvey, I., Willoughby, I. and Frazier, R.A., (2007) Hydrolyzable tannin structures influence relative globular and random coil protein binding strengths. J. Agric. Food Chem., 55(11): 4554-4561.

49. González, S., Pabon, M.L. and Carulla, J. (2005) Effects of tannins on in vitro ammonia release and dry matter degradation of soybean meal. Arch. Latinoam Prod. Anim., 10: $97-101$

50. Bhatta, R., Mani, S., Baruah, L. and Sampath, K.T. (2012) Phenolic composition, fermentation profile, protozoa population and methane production from sheanut (Butryospermum parkii) byproducts in vitro. Asian Australas. J. Anim. Sci., 25(10): 1389-1394.

51. Mamat, S.S., Kamarolzaman, M.F.F., Yahya, F., Mahmood, N.D., Shahril, M.S., Jakius, K.F., Mohtarrudin, N., Ching, S.M., Susanti, D., Taher, M. and Zakaria, Z.A. (2013) Methanol extract of Melastoma malabathricum leaves exerted antioxidant and liver protective activity in rats. BMC Complement. Altern. Med., 13(1): 326.

52. Oskoueian, E., Abdullah, N. and Oskoueian, A. (2013) Effects of flavonoids on rumen fermentation activity, methane production, and microbial population. BioMed. Res. Int., 2013: 349129.

53. Kim, E.T., Le Luo Guan, S.J.L., Lee, S.M., Lee, S.S., Lee, I.D., Lee, S.K. and Lee, S.S. (2015) Effects of flavonoid-rich plant extracts on in vitro ruminal methanogenesis, microbial populations and fermentation characteristics. Asian Australas. J. Anim. Sci., 28(4): 530.

54. Goel, G., Makkar, H.P.S. and Becker, K. (2008) Changes in microbial community structure, methanogenesis and rumen fermentation in response to saponin-rich fractions from different plant materials. J. Appl. Microbiol., 105(3): 770-777.

55. Goel, G. and Makkar, H.P. (2012) Methane mitigation from ruminants using tannins and saponins. Trop. Anim. Health Prod., 44(4): 729-739. 